\title{
Prof. Dr. Luther W. Brady verstorben
}

\section{Hans-Peter Heilmann ${ }^{1}$}

Online publiziert: 30. August 2018

(c) Springer-Verlag GmbH Germany, part of Springer Nature 2018

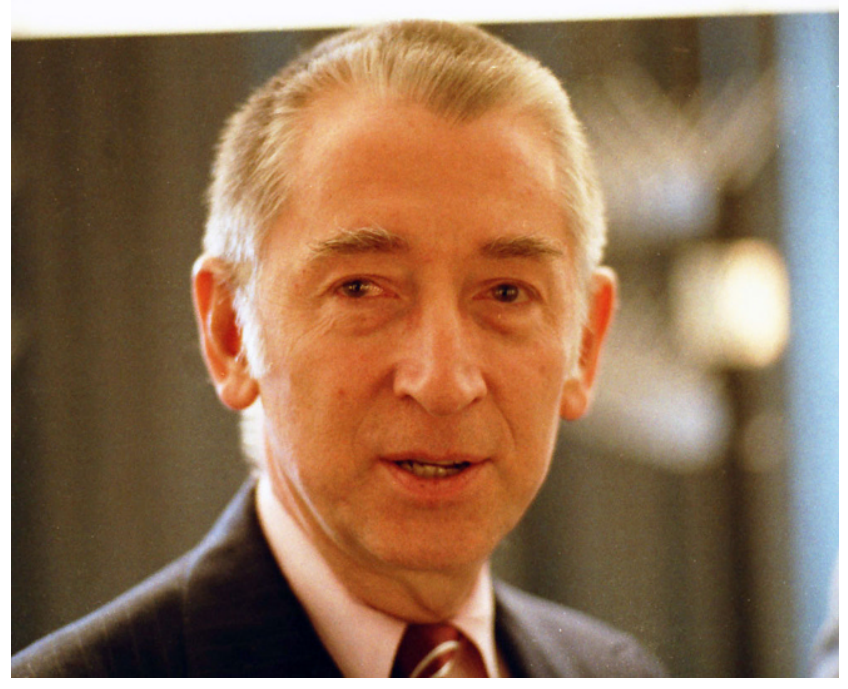

Luther W. Brady

Luther Brady wurde am 20.10.1925 in Rocky Mount, North Carolina, geboren. Er besuchte die Highschool in Wilson, North Carolina, machte zunächst einen Bachelor in Zoologie und anschließend den Medical Doctor (MD) 1948 an der Washington University.

Es folgten sechs Monate an der Abteilung für Pathologie des National Cancer Institute. Danach verbrachte er die Internship am Jefferson Medical College Hospital in Philadelphia.

Während des Korea-Krieges diente er in der United States Navy. Hier wurde er vom Commanding Officer gefragt, ob er das Management des Nuklearmedizin-Programms am Naval Hospital in Bethesda übernehmen könne. Er leitete das nuklearmedizinische Programm für ein Jahr und blieb dann bis zum Ende seiner Militärzeit auf einem Kreuzer, der von Yokosuka (Japan) aus operierte.

Prof. Dr. med. Hans-Peter Heilmann

hp.heilmann@gmx.de

1 Alte Landstr. 220, 22391 Hamburg, Deutschland
Nach Entlassung aus der Navy kehrte er an das Jefferson Medical College Hospital zurück. Er begann die Residency in Radiologie und leitete gleichzeitig das nuklearmedizinische Programm. Während dieser Zeit erwachte sein Interesse an der Strahlentherapie, sodass er die Weiterbildung im Programm der „radiation oncology“ unter Dr. Th. Eberhardt fortsetzte. Im Juni 1955 wechselte er an das Hospital der Universität von Pennsylvania zu Dr. Eugene P. Pendergrass, wo er schließlich Mitglied der Fakultät „Radiation Oncology" an der Universität von Pennsylvania wurde.

Im Juli 1959 wurde Brady Assistant Professor am Department of Radiology des Columbia University College in New York City, 1963 Professor. 1970 übernahm er die Position des Chefs des „Radiation Oncology Department“ am Hahnemann Medical College and Hospital in Philadelphia. 1975 wurde er zum Hylda Cohn Professor für klinische Onkologie der American Cancer Society ernannt.

Nach dem Jefferson Medical College unter Leitung von Dr. Simon Kramer wurde die Strahlentherapie am Hahnemann Medical College die zweite unabhängige Abteilung für Strahlentherapie in den USA.

In den frühen 50er Jahren wurde unter dem Einfluss von Dr. Juan del Regato der American Club of Therapeutic Radiologists gegründet, dessen 1. Präsident Dr. Simon Kramer war. Brady war Gründungsmitglied. Aus diesem Club ging später die American Society of Therapeutic Radiology and Onkology (ASTRO) hervor.

Brady war einer der führenden Radioonkologen der USA. In diversen amerikanischen, europäischen und internationalen Fachgesellschaften war er nicht nur Mitglied, sondern bekleidete Vorstandsämter und wurde Ehrenmitglied. Er erhielt 24 Ehrenmedaillen, davon 11 von amerikanischen Gesellschaften, darunter mehrere Goldmedaillen.

Aus deutscher Sicht sind die Ehrenmitgliedschaft in der Deutschen Röntgengesellschaft (DRG) und in der Deutschen Gesellschaft für Radioonkologie (DEGRO) zu erwähnen. Neben zwei amerikanischen Ehrendoktorwürden war er Ehrendoktor der Universität Heidelberg und der Universität Göttingen.

Sein Einfluss auf junge Radioonkologen in den USA, aber auch in Europa kann gar nicht hoch genug eingeschätzt 
werden. Für die jüngeren Kollegen aus Deutschland waren Luther Brady und andere väterliche Freunde, die ihnen den Zugang zu amerikanischen Universitäten und Gesellschaften erleichterten.

Bradys wissenschaftliches Werk umfasst u.a. 550 Originalarbeiten. Er war mit Carlos Perez Herausgeber des Standardlehrbuchs „Principles and Practice of Radiation Oncology“ und mit Theodore E. Yaeger Herausgeber der „Encyclopedia of Radiation Oncology“. Seit 1977 gab er die Zeitschrift „American Journal of Clinical Oncology“ heraus. Darüber hinaus war er Co-Editor in 11 weiteren Fachzeitschriften. Als Meilenstein wird seine Publikation „Radiation Oncology - Programs for the Present and Future" aus dem Jahr 1985 betrachtet.

Zusammen mit M. W. Donner, H.-P. Heilmann, F. Heuck, H. Molls und C. Nieder war er seit 1984 Herausgeber der Reihe „Medical Radiology. Radiation Oncology“ im Springer-Verlag, der Nachfolge des Handbuchs für Radiologie.

Den Lehrstuhl an der Hahnemann-Universität gab Brady in den späten 90er Jahren aus Altersgründen ab, blieb aber klinisch und wissenschaftlich weiter tätig. 2006 gründete er das Philadelphia CyberKnife Zentrum.

Manche seiner Freunde bezeichneten Brady als einen Renaissancemenschen. Sein Interesse galt nicht nur der Medi- zin, sondern auch der bildenden Kunst, der Musik und der Oper. Mit dem Künstler Sir Gordon Howard Eliot Hodgkin war er befreundet. Er besaß eine große Anzahl von Bildern sowohl dieses modernen Malers als auch anderer zeitgenössischer Künstler. Viele seiner Bilder sind in der „Luther W. Brady Art Gallery“ der George Washington University ausgestellt. Er war u.a. Sponsor des Philadelphia Museum of Art.

Aufgrund seiner Liebe zur klassischen Musik unterstützte er aktiv das Philadelphia Orchester, sein Interesse für die Oper zeigte sich an Mitgliedschaften in der Metropolitan Opera, New York City, und der Santa Fe Opera.

Luther W. Brady ist am 13. Juli 2018 im Alter von fast 93 Jahren an Herzversagen gestorben.

Einen so unglaublich vielseitigen Lebenslauf findet man selten. Anlässlich der Verleihung einer Goldmedaille sagte der amerikanische Radiologe James Cox:

Ein so außergewöhnlicher Mensch mit so unzähligen Qualitäten muss aber letztlich irgendwo auch eine Schwachstelle haben. Es hat mich viel Mühe und Zeit gekostet, aber ich habe sie gefunden:

„His backhand is not so good“.

Prof. Dr. med. Hans-Peter Heilmann 